\title{
Haptic Guidance in Dynamic Environments Using Optimal Reciprocal Collision Avoidance
}

\author{
Tommaso Lisini Baldi ${ }^{1,2}$, Stefano Scheggi ${ }^{1}$, Marco Aggravi ${ }^{1}$, and Domenico Prattichizzo ${ }^{1,2}$
}

\begin{abstract}
Human guidance in situations where the users cannot rely on their main sensory modalities, such as assistive or search-and-rescue scenarios, is a challenging task. In this paper, we address the problem of guiding users along collision-free paths in dynamic environments, assuming that they cannot rely on their main sensory modalities. In order to safely guide the subjects, we adapt the Optimal Reciprocal Collision Avoidance to our specific problem. The proposed algorithm takes into account the stimuli which can be displayed to the users and the motion uncertainty of the users when reacting to the provided stimuli. The proposed algorithm was evaluated in three different dynamic scenarios. A total of 18 blindfolded human subjects were asked to follow haptic cues in order to reach a target area while avoiding real static obstacles and moving users. Three metrics such as time to reach the goal, length of the trajectories, and minimal distance from the obstacles are considered to compare results obtained using this approach and experiments performed without visual impairments. Experimental results reveal that blindfolded subjects are successfully able to avoid collisions and safely reach the targets in all the performed trials. Although in this paper we display directional cues via haptic stimuli, we believe that the proposed approach can be general and tuned to work with different haptic interfaces and/or feedback modalities.
\end{abstract}

Index Terms-Haptics and Haptic Interfaces, Collision Avoidance, Human Guidance

\section{INTRODUCTION}

$\mathbf{L}$ ET us consider the problem of guiding a subject toward a goal location in a dynamic environment while avoiding obstacle collisions (Fig. 1). Possible scenarios are assistive and search-and-rescue scenarios. In such cases, environmental noise, dust, or fog from debris severely reduce the human operator sensing. Other examples of applicability of human guidance are human-robot cooperative tasks, where the robot can guide the user along collision-free paths without violating the mechanical constraints of the robot itself.

Manuscript received: February, 15, 2017; Revised May, 04, 2017; Accepted July, 15, 2017.

This paper was recommended for publication by Yasuyoshi Yokokohji upon evaluation of the Associate Editor and Reviewers' comments. The research leading to these results has received funding from the European Union Seventh Framework Programme FP7/2007-2013 under grant agreement n. 601165 of the project "WEARHAP - WEARable HAPtics for humans and robots" and from the European Union's Horizon 2020 research and innovation programme - Societal Challenge 1 (DG CONNECT/H) under grant agreement n. 643644 of the project "ACANTO: A CyberphysicAl social NeTwOrk using robot friends".

${ }^{1}$ Tommaso Lisini Baldi, Stefano Scheggi, Marco Aggravi, and Domenico Prattichizzo are with the Department of Information Engineering and Mathematics, University of Siena, Via Roma 56, I-53100 Siena, Italy. \{lisini, scheggi, aggravi, prattichizzo\}@diism.unisi.it

${ }^{2}$ Tommaso Lisini Baldi and Domenico Prattichizzo are with the Department of Advanced Robotics, Istituto Italiano di Tecnologia, Genova, 16163, Italy. \{tommaso.lisini, domenico.prattichizzo\}@iit.it

Digital Object Identifier (DOI): see top of this page.

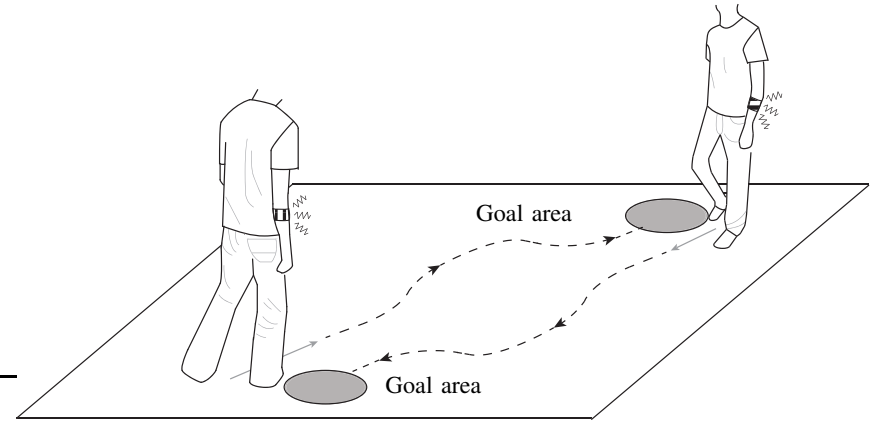

Fig. 1. In this paper, we address the problem of guiding human subjects in situations where the users cannot rely on their main sensory modalities. The subjects have to reach the respective goal areas while avoiding collisions with static obstacles and moving users. The proposed obstacle avoidance policy generates online suitable stimuli (in our specific case, haptic stimuli), which guide the users along collision-free trajectories (dotted).

Over the years, haptic feedback has been found an effective, yet non-intrusive way for providing directional cues to users. It represents an interesting way to provide information when audio and visual modalities are not available. In fact, audio and/or visual displays may be ineffective in circumstances where vision is temporarily impaired. In search-and-rescue scenarios, background noise can make auditory feedback difficult to hear or understand. Regarding the haptic feedback, most of the existing research focused on providing cutaneous stimulation mainly via bracelets and waist belts.

A vibrotactile waist belt composed of eight tactors was used for waypoint navigation in outdoor scenarios [1]. The waist belt displayed both the direction and distance to the next waypoint. A similar device was used to provide vibrotactile cues for improving situational awareness of soldiers in a simulated building-clearing exercise [2]. In [3], a vibrotactile belt was used for human guidance in indoor environments. Continuous vibrotactile stimuli were used to display directional and rotational motions to the blindfolded users. A path planning algorithm generated suitable directional clues to to avoid collisions with static and dynamic obstacles. Vibrotactile armbands were used to navigate subjects along fixed paths using three haptic stimuli: Turn left, turn right, slow down, [4]. No dynamic obstacles were considered by the authors. Similar devices and haptic policy were used to guide blindfolded users in dynamic environments assisted by a mobile robot [5], [6]. The robot recomputed the desired path as soon as new obstacles (static/dynamic) were detected. With regard to haptic stimuli for blind navigation, an indoor localization system was presented in [7]. The system used Bluetooth for localization, and provided surrounding environment information by means of five tactors placed on the chest and on the shoulders of 
the users. Three haptic stimuli were considered: Go straight, turn left, and turn right. In [8], the authors developed an electronic bracelet which provided vibrations when an obstacle was close to the user. The vibration magnitude was directly proportional to the obstacle distance. Waypoint navigation via haptic feedback was exploited in [9], where the authors evaluated the use of haptic stimuli for indoors pedestrian guidance using two wrist-worn interfaces. Finally, in [10], a comparison of different vibrotactile devices for guiding visually impaired people was proposed.

Recently, path-planning algorithms for humans were proposed in [11], [12]. In [11], the authors proposed a pathplanning algorithm based on Rapidly-exploring Random Trees (RRT*). The algorithm allows one user to reach a goal area while always keeping in contact with other humans in the surroundings using a Software Defined Radio. In [12], the authors designed a path panning solution for mobile robots to assist navigation for older adults and disabled users. The proposed method selected the optimal path by taking into account the user comfort in performing it.

In this paper, we present a human navigation policy to guide multiple users along collision-free paths in dynamic environments. For each subject, the proposed navigation policy generates online suitable directional cues in order to minimize the possibility of collisions among the users, while avoiding as much as possible oscillations in the users' motions. The proposed method relies on the Optimal Reciprocal Collision Avoidance (ORCA) algorithm for non-holonomic agents proposed in [13], that we adapt to our specific problem. ORCA has been demonstrated to provide smooth, collision-free motions which are as close as possible to the desired motions of the agents. The proposed algorithm is based on the assumption that the human locomotion can be approximated by the motion of a unicycle system, i.e., non-holonomic constraints similar to those of mobile robots seem to be at work when a human is walking [14]. In designing the proposed obstacle-avoidance algorithm, we address the following challenges. First, it is worth pointing out that while it is simple to steer a robot, it is not trivial to impose a desired velocity to a human. In fact, by providing directional cues via haptic feedback, only a discrete set of different stimuli (i.e., instructions) can be displayed to the users. Such set of stimuli is far smaller than the set of all the possible velocities that a user can perform. Moreover, the larger is the set of stimuli provided to the users, the harder could be for a subject to recognize a particular stimulus and to react accordingly. Second, when a user perceives a guiding stimulus, she/he will never react in the same exact way. Different from related studies, the proposed algorithm takes into account the limited number of stimuli that can be displayed to the users, and the motion uncertainty of the users when reacting to a particular stimulus.

We evaluate the proposed obstacle avoidance algorithm in combination with haptic stimuli. The haptic policy has been demonstrated to be intuitive and effective in guiding users in mixed human-robot scenarios [15], [5], [6], older adults in assistive tasks [16], and visually impaired [17]. Without loss of generality, in what follows we assume that the human is free to select her/his desired walking speed. Control signals

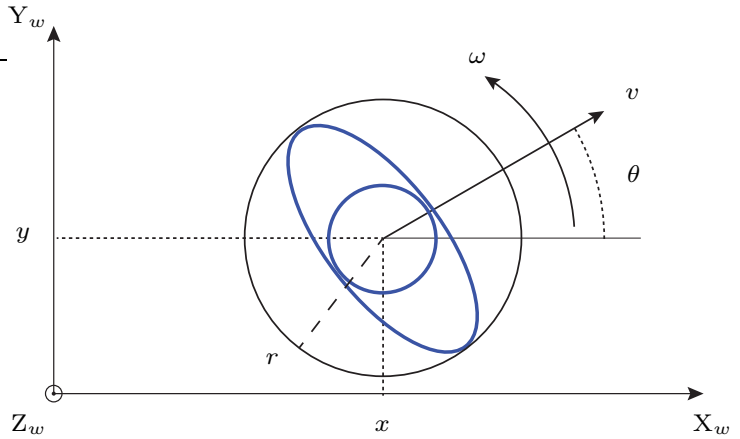

Fig. 2. The proposed approach assumes that the human locomotion can be approximated by the motion of a unicycle system. $[x, y]^{T}$ and $\theta$ represent the position and orientation of the human w.r.t. the world reference frame $\left\langle\mathrm{O}_{w}, \mathrm{X}_{w}, \mathrm{Y}_{w}, \mathrm{Z}_{w}\right\rangle$ and $v, \omega$ represent the linear and angular velocities of the user. In the proposed setup, the human is free to select her/his desired linear velocity while control signals are sent to the human to steer her/his locomotion by acting on $\omega$. Every user (navy blue) is modeled as as discshaped entity having radius $r$.

(i.e., haptic stimuli) are sent to the users in order to steer their locomotion. The proposed method is evaluated in three different scenarios consisting of: ( $i$ ) Two users; ( $i$ i) two users and a static obstacle; and (iii) three users. A total of 18 users participate in the evaluation. In all scenarios, the users have to move toward their respective goal areas, while avoiding reciprocal collisions and collisions with the environment. Three metrics such as time to reach the goal, length of the trajectories, and minimal distance from the agents have been considered to compare the results obtained using this approach with experiments performed with sighted people.

The proposed research demonstrates the navigation of multiple users in dynamic scenarios, assuming that: $(i)$ The users cannot rely on their main sensory modalities; (ii) a limited discrete set of directional cues can be displayed to the users. Different from related research, the users do not rely on additional tools (white cane). Moreover, the proposed approach tries to avoid as much as possible oscillations in the users' motions. Although in this work we display directional cues via vibrotactile stimuli, the proposed approach can be general and tuned to work with different haptic interfaces and/or feedback modalities (audio, visual).

The rest of the paper is organized as follows. Sect. II presents the obstacle avoidance policy used in our problem. Sect. III reviews the model of human locomotion and the proposed vibrotactile haptic policy. In Sect. IV, we validate the proposed algorithms via real-world experiments, whereas in Sect. V, results are given and discussed. Finally, in Sect. VI we summarize the main contributions of the paper, and we discuss possible avenues for future research.

\section{OBSTACLE AVOIDANCE FOR HUMAN NAVIGATION}

In this section, we describe the obstacle avoidance algorithm used to safely navigate the users in dynamic environments. The proposed algorithm is based on the assumption that the human locomotion can be approximated by the motion of a unicycle system, i.e., the human's walking direction is tangential to her/his trajectory [14]. Let $\mathbf{x}=[x, y, \theta]^{T}$ be the pose of a user whose kinematics can be abstracted as a unicycle 


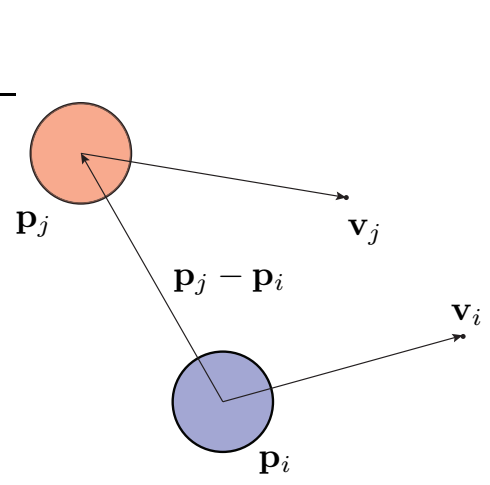

(a)

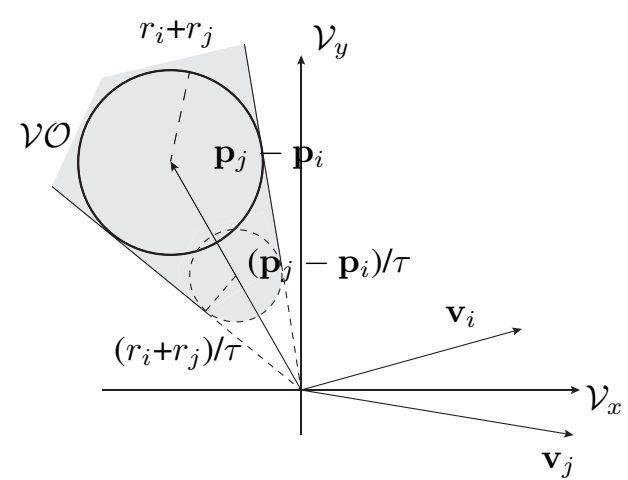

(b)

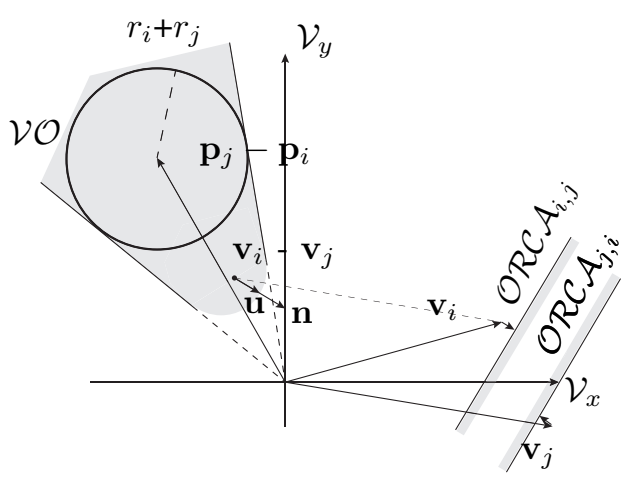

(c)

Fig. 3. (a) Let us assume a configuration with two holonomic agents on a collision course. (b) The unsafe region (Velocity Obstacle $(\mathcal{V O})$ ) in the velocity space for agent $i$ induced by agent $j$ is a truncated cone built by considering the half-lines emanating from the origin, tangent to a disk at the relative position of the two agents having radius $r_{i}+r_{j}$. The amount of truncation depends on the time window $\tau$. $\mathcal{V O}$ is the set of all relative velocities of agent $i$ with respect to agent $j$ that will result in a collision between $i$ and $j$ at some moment $\tau$. (c) The set of permitted velocities for agent $i$ for optimal reciprocal collision avoidance with a user $j$ is a half-plane delimited by the line perpendicular to $\mathbf{u}$ through the point $\mathbf{v}_{i}+1 / 2 \mathbf{u}$, where $\mathbf{u}$ is the vector from $\mathbf{v}_{i}-\mathbf{v}_{j}$ to the closest point on the boundary of the $\mathcal{V O}$. u is the smallest change required to the relative velocity of agent $i$ and $j$ to avoid collision within $\tau$ time. The agents share the responsibility of avoiding collisions among them, i.e., agent $i$ adapts its velocity by (at least) $1 / 2 \mathbf{u}$ and assumes that agent $j$ takes care of the remaining half. Collision-free velocities for agent $i$ are the velocities which satisfy constraint $\mathcal{O} \mathcal{R C} \mathcal{A}_{i, j}\left(\right.$ right side of the half-plane defined by $\mathcal{O} \mathcal{R} \mathcal{C} \mathcal{A}_{i, j}$ ), while collision-free velocities for agent $j$ are the velocities which satisfy constraint $\mathcal{O R C} \mathcal{A}_{j, i}\left(\right.$ left side of the half-plane defined by $\mathcal{O} \mathcal{C} \mathcal{A}_{j, i}$ ).

model, where $\mathbf{p}=[x, y]^{T}$ and $\theta$ represent the position and orientation of the human w.r.t. the world reference frame $\left\langle\mathrm{O}_{w}, \mathrm{X}_{w}, \mathrm{Y}_{w}, \mathrm{Z}_{w}\right\rangle$, respectively (see Fig. 2). Let $v, \omega$ be the linear and angular velocities of the human. The dynamic model of the human motion can be described as,

$$
\dot{\mathbf{x}}=\left[\begin{array}{c}
\cos (\theta) \\
\sin (\theta) \\
0
\end{array}\right] v+\left[\begin{array}{l}
0 \\
0 \\
1
\end{array}\right] \omega
$$

We assume that the agents (in our case the humans) are modeled as disc-shaped entities having radius $r_{i}, i=1, \ldots, n$, being $n$ the number of agents. The algorithm is based on the extension of the Optimal Reciprocal Collision Avoidance (ORCA) algorithm for non-holonomic robots (NH-ORCA) presented in [13], that we adapt to our specific problem.

ORCA is a velocity-based collision avoidance approach for multiple holonomic agents (Fig. 3), [18]. The algorithm provides a sufficient condition for each agent to be collisionfree for at least a fixed amount of time $\tau$ into the future. Each agent takes into account the observed velocity and pose of the other agents in order to avoid collisions with them. Then, the optimal velocity is selected by using linear programming. The main advantage of ORCA with respect to other obstacle avoidance algorithms is that it provides smooth, collisionfree motions, avoiding as much as possible oscillations in the agents' paths.

NH-ORCA is the generalized version of ORCA for any non-holonomic agents. The underlying idea is that any nonholonomic agent $i$ can track a holonomic speed vector $\mathbf{v}_{i}$ with a certain tracking error $\epsilon_{i}$, i.e., a non-holonomic robot can drive along an arc and then along a straight line which is close to a holonomic vector in that direction (Fig. 4). In accordance with [13] we can compute the holonomic speed vector $\mathbf{v}_{i}$ that approximates the non-holonomic velocity with the minimum error $\epsilon_{i}$ as follows,

$$
\mathbf{v}_{i}=v_{i, h}\left[\cos \left(\theta_{i, h}\right), \sin \left(\theta_{i, h}\right)\right]^{T}
$$

If $\omega_{i} \neq 0$, then (2) can be computed assuming,

$$
\theta_{i, h}=\omega_{i} t \quad v_{i, h}=v_{i} \frac{2\left(1-\cos \left(\omega_{i} t\right)\right)}{\omega_{i} t \sin \left(\omega_{i} t\right)},
$$

where $\omega_{i}$ is the non-holonomic angular velocity, and $t \neq 0$ is the time to achieve the correct orientation $\theta_{i, h}$. If $\omega_{i}=0, \mathbf{v}_{i}$ can be computed from (2) assuming $v_{i, h}=v_{i}$ and $\theta_{i, h}=0$. Given a non-holonomic velocity $\left(v_{i}, \omega_{i}\right)$ with $\omega_{i} \neq 0$, the maximum error $\epsilon_{i}$ in tracking the related holonomic velocity $\mathbf{v}_{i}$ is given as (cf. [13]),

$$
\begin{aligned}
& \epsilon_{i}^{2}\left(v_{i}, \omega_{i}, v_{i, h}, \theta_{i, h}\right)= \\
& \quad v_{i, h}^{2} t^{2}-\frac{2 v_{i, h} t \sin \left(\theta_{i, h}\right)}{\omega_{i}} v_{i}+\frac{2\left(1-\cos \left(\theta_{i, h}\right)\right)}{\omega_{i}^{2}} v_{i}^{2} .
\end{aligned}
$$

NH-ORCA consists in the following steps. First, a set of allowed holonomic velocities is calculated based on the current non-holonomic velocities and maximum tracking error $\epsilon_{i}$. Resulting constraints are computed and added to the linear program in the ORCA formulation. To allow collision-free navigation, $r_{i}$ has to be increased by the tracking error $\epsilon_{i}$, since the agents do not track the desired holonomic velocity exactly. Finally, the desired holonomic velocity is computed via linear programming and mapped to the corresponding non-holonomic control inputs, which guarantee collision-free motions. Velocity-based collision avoidance approaches use the pose of the agents and their actual velocity to generate collision-free velocities [13], [18], [19]. Guiding users via haptic, audio, or visual stimuli, implies that we are not imposing a desired velocity to the subjects (different from a robot). Instead, we are providing stimuli which should be translated into suitable velocities. This arises two challenges. First, a mapping between the directional stimuli and the velocity of the human should be defined. Second, motion uncertainty of the users when reacting to a given stimulus should be taken into account (cf. Sect. I).

For the mapping between the directional stimuli and the velocities of the human, we perform an offline procedure which consists in sending directional stimuli to the users while 
walking and analyzing their behaviors (cf. Sect. III). The online obstacle avoidance algorithm consists in the following steps. Let $\delta t$ be the constant sampling time of the system and let $N_{S}$ be the number of stimuli displayed to the users. At each iteration, the proposed collision avoidance policy performs a continuous cycle of sensing and acting for each user (Fig. 5). First, the system estimates the pose and actual velocity of all the users. For each user $i$, the algorithm calculates the holonomic velocities $\mathbf{v}_{i}$ and related tracking errors $\epsilon_{i}$ from the actual non-holonomic velocities $\left(v_{i}, \omega_{i}\right)$, (Fig. 5(a)). By using the holonomic velocities $\mathbf{v}_{i}$ and the tracking errors $\epsilon_{i}$, constraints are added to the linear program in the ORCA formulation (Fig. 5(b)). Each constraint is represented as a half plane in the holonomic velocity space. Let $\mathbf{p}_{i}=\mathcal{N}\left(\mu_{\mathbf{p}_{i}}, \Sigma_{\mathbf{p}_{i}}\right)$ be a bivariate normal distribution of the measured position $\mathbf{p}_{i}$ of the user $i$, having mean $\mu_{\mathbf{p}_{i}}$ and standard deviation $\Sigma_{\mathbf{p}_{i}}=\operatorname{diag}\left(\sigma_{\mathbf{p}_{i}}, \sigma_{\mathbf{p}_{i}}\right)$. For example, positions might be estimated using an Extended Kalman Filter which provides an estimate of the variance, and hence the standard deviation, of the measured quantities. These values are taken into account by the obstacle avoidance algorithm by expanding the edges of the velocity obstacle (Fig. 5(c)). In order to select the guiding stimulus $k=1, \ldots, N_{S}$, first we calculate the holonomic velocities $\Sigma_{S_{k}}$ related to the non-holonomic velocities and uncertainty of stimulus $k$ (Fig. 5(c)). Finally, the algorithm selects the stimulus whose $\Sigma_{S_{k}}$ maximizes the intersection with the obstacle-free region in the ORCA formulation. It is worth pointing out that the collision-free velocities are computed in order to be as close as possible to the preferred ones. In our particular case, the preferred velocities are the ones that minimize the walking time of the users towards their goal areas. The proposed algorithm differs from [13], since NH-ORCA starts by considering a holonomic behavior for the agent. Then, a set of holonomic allowed velocities is computed. Finally, the algorithm calculates the optimal holonomic velocity, which is mapped to the corresponding non-holonomic control inputs for the agent. Moreover, the proposed algorithm takes into account the fact that only a discrete set of stimuli (i.e., control inputs) can be displayed to a user and the presence of motion uncertainty when the users react to such stimuli.

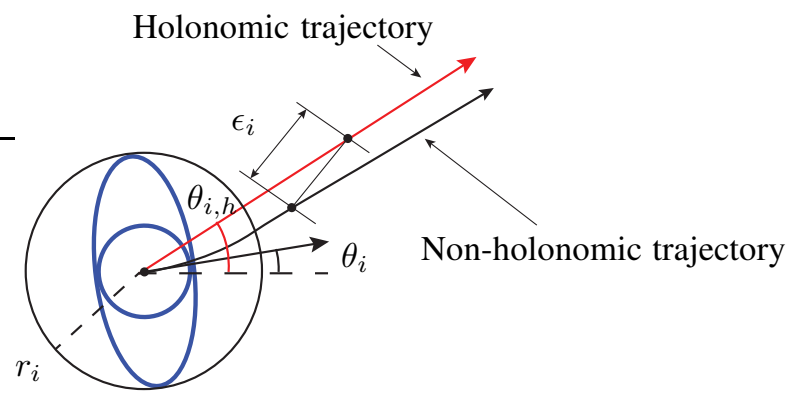

Fig. 4. The underlying idea of NH-ORCA is that any non-holonomic agent $i$ can track a holonomic speed vector with a certain tracking error $\epsilon_{i}$. Such error $\epsilon_{i}$ is used to compute the collision-free velocity based on the actual pose and velocity of the user.

\section{HUMAN GUIDANCE VIA HAPTIC FEEDBACK}

In this section, we briefly describe the haptic guidance policy used to validate the obstacle avoidance algorithm described in Sect. II. The proposed policy is based on the assumption that the human locomotion can be approximated by the motion of a unicycle system (cf. Sect. II). Moreover, we assume that the human is free to select her/his desired walking speed. Thus, haptic stimuli are sent to the user in order to steer her/his heading.

Let us consider the problem of guiding a user along a path, given her/his walking pace. This problem consists in steering the human by acting on her/his angular velocity $\omega$. In order to provide stimuli which are easily recognizable by the user, the device could elicit only three basic behaviors on the human (turn left, turn right, and go straight). Thus, only three stimuli would be sufficient in principle. As a consequence, we display vibrotactile stimuli via two haptic armbands placed on the forearms: vibration of the left armband alerts the participant to turn left $(L)$, while vibration of the right armband alerts the participant to turn right $(R)$. If the armbands do not vibrate, it means that the user can go straight $(C)$. Each armband is made by two motors with an effective vibration frequency range of $100-280 \mathrm{~Hz}$ (the maximal sensitivity is achieved around 200$300 \mathrm{~Hz},[20])$, as depicted in Fig. 6. In order to not overload the user's tactile channel and to not reduce the recognition time, we do not modulate the frequency of the signal but we use a simple on/off mechanism, similar to [5]. We used an additional stimulus $(S)$ to stop the user by activating both the haptic devices. When an armband is engaged, its motors alternatively vibrate for $0.2 \mathrm{~s}$ at a frequency of $250 \mathrm{~Hz}$.

In order to define a relationship between the haptic stimuli and the velocity of the users, let us remind that we are interested in steering the users by changing their angular velocity $\omega$ (cf. Eq. (1)). Since three stimuli (turn left, turn right, and go straight) have been demonstrated to be intuitive and effective in guiding users along path [4], it is necessary to find the relationship between the proposed three stimuli and the angular velocity of the users. That is, we need to find the amount of angular velocity that the users apply when they perceive the proposed haptic stimuli. Preliminary experiments were conducted on 20 blindfolded subjects (17 males, age range 23-40, 19 right-handed). Participants were instructed to walk along a walkway, of about $4 \mathrm{~m}$, whilst wearing the armbands and to react accordingly to the stimulus type, as soon as they perceive it. The stimulus was sent as soon as the users have walked for $1 \mathrm{~m}$. The armband continued to vibrate for $2 \mathrm{~s}$ after the activation. For each stimulus type, every subject performed 12 trials, organized in a pseudo-random order. All subjects were blindfolded and wore circumaural headphones, reproducing white noise to mask distracting ambient or cuing

TABLE I

MEAN AND STANDARD DEVIATION OF THE ANGULAR VELOCITY WHEN THE PROPOSED STIMULI ARE DISPLAYED TO THE USERS

\begin{tabular}{c|c} 
Haptic stimulus & $\omega(\mathrm{rad} / \mathrm{s})$ \\
\hline Turn left & $1.08 \pm 0.31$ \\
Turn right & $-1.11 \pm 0.33$ \\
Go straight & $0 \pm 0.11$
\end{tabular}




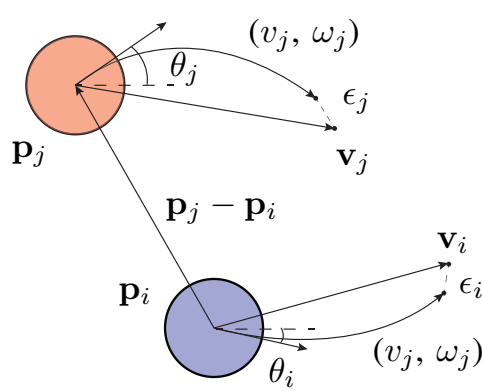

(a)

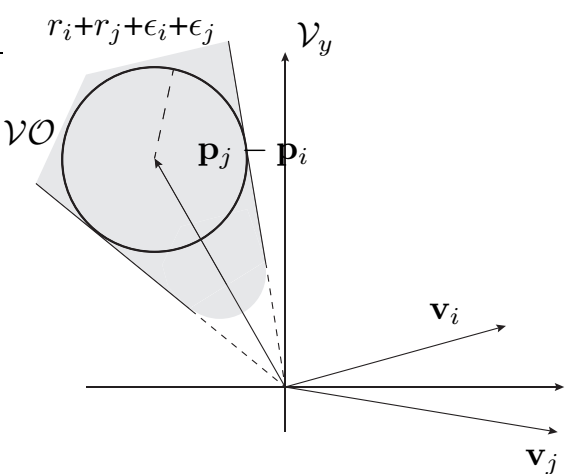

(b)

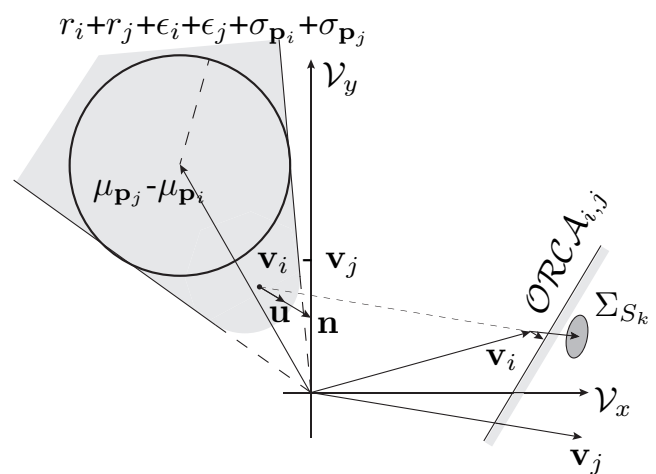

(c)

Fig. 5. (a) Let us assume a configuration with two disc-shaped non-holonomic agents on a collision course. (b) In order to determine the Velocity Obstacle $\mathcal{V O}$ in the velocity space, we first compute the holonomic velocity $\mathbf{v}_{i}, \mathbf{v}_{j}$ from the non-holonomic ones $\left(v_{i}, \omega_{i}\right),\left(v_{j}, \omega_{j}\right)$. The $\mathcal{V O}(\operatorname{shaded}$ area) is a truncated cone tangent to a disk at the relative position of the two agents having radius $r_{i}+r_{j}+\epsilon_{i}+\epsilon_{j}$, being $\epsilon_{i}, \epsilon_{j}$ the errors the agents will perform in tracking holonomic velocities. (c) If the positions of the agents are obtained from noisy measurements, (i.e., they are drawn form normal distributions $\left.\mathbf{p}_{i}=\mathcal{N}\left(\mu_{\mathbf{p}_{i}}, \Sigma_{\mathbf{p}_{i}}\right), \Sigma_{\mathbf{p}_{i}}=\operatorname{diag}\left(\sigma_{\mathbf{p}_{i}}, \sigma_{\mathbf{p}_{i}}\right) \mathbf{p}_{j}=\mathcal{N}\left(\mu_{\mathbf{p}_{j}}, \Sigma_{\mathbf{p}_{j}}\right)\right), \Sigma_{\mathbf{p}_{j}}=\operatorname{diag}\left(\sigma_{\mathbf{p}_{j}}, \sigma_{\mathbf{p}_{j}}\right)$ the $\mathcal{V O}$ is updated accordingly. The algorithm selects the stimulus $k=1, \ldots, N_{S}$ whose holonomic velocity and related uncertainty $\Sigma_{S_{k}}$ maximize the intersection with the collision-free region. For agent $i$, the desired stimulus should maximize the intersection of $\Sigma_{S_{k}}$ with the region delimited by $\mathcal{O} \mathcal{R} \mathcal{A}_{i, j}$.

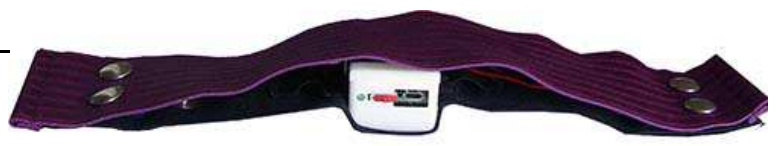

(a)

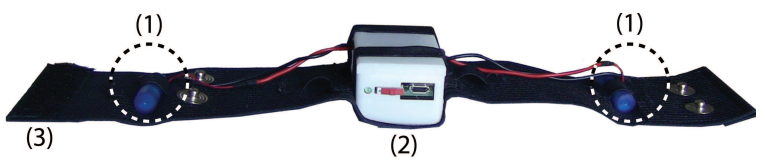

(b)

Fig. 6. Directional cues are provided to the users via two vibrotactile armbands placed on the forearms. The haptic armbands are composed of two vibrating motors (1) attached to an elastic wristband (3). A Li-Ion battery and an Arduino board are in (2).

sounds from the armbands. Human motion was tracked via an optical tracking system (Vicon Motion Systems Ltd, Oxford, UK). All the subjects correctly reacted to the haptic stimuli. Table I reports the mean and standard deviation of the angular velocity when the proposed stimuli were displayed to the users.

Algorithm 1 describes the proposed collision avoidance method when coupled with haptic stimuli for human guidance. For simplicity, in the algorithm we consider possible collisions among users. However, the proposed approach can be easily extended to avoid collisions with static objects such as walls, etc. In this case, the ORCA half-plane for user $i$ is computed at the point $\mathbf{v}_{i}+\mathbf{u}$ instead of at point $\mathbf{v}_{i}+1 / 2 \mathbf{u}$ (Fig. 3).

\section{EXPERIMENTAL VALIDATION}

In this section, we present an experimental evaluation performed to assess the feasibility and functionality of our approach. We validated the proposed approach in three different scenarios. In the first, two users were asked to reach two different goal areas, wearing two vibrotactile armbands each (one per arm), which displayed the directional cues. We defined this scenario as S1. In the second scenario, S1 was augmented by introducing a static obstacle. Both users still had to reach two different goal areas, while avoiding the object.
Algorithm 1 Optimal Reciprocal Collision Avoidance for human guidance via haptic feedback. Let $N_{U}$ be the number of users and let $N_{S}=3$ be the number of haptic stimuli which can be displayed to the users (turn left, turn right, and go straight). For each agent $i=1, \ldots, N_{U}$ the algorithm computes the ORCA constraints (region ORCA $_{\text {) with respect }}$ to all the other agents. The algorithm considers the position $\mathbf{p}_{i}$, the holonomic velocity $\mathbf{v}_{i}$, and velocity approximation error $\epsilon_{i}$ of the agent. Then, a numerical method is used to compute desired haptic stimulus which maximizes the intersection of the collision-free region defined by the ORCA constraint and the region $\Sigma_{k}$ (defined by the velocity and uncertainty related to the haptic stimulus $k=1, \ldots, N_{S}$ ).

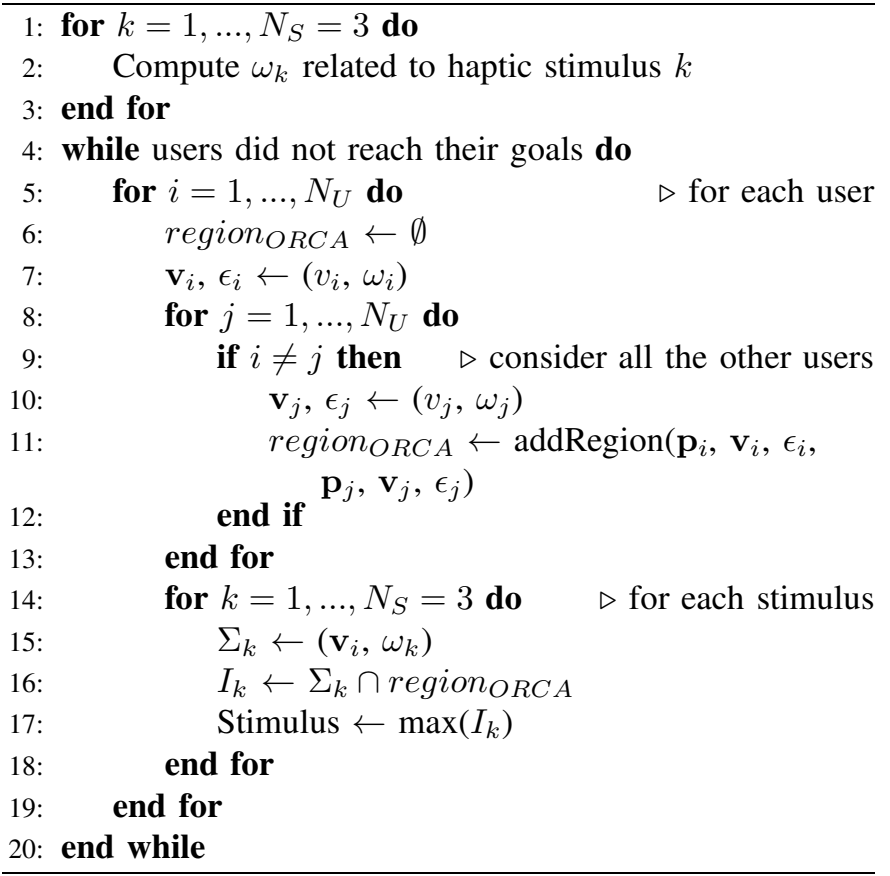

This scenario was named S2. The third scenario, named S3, was built by taking $\mathrm{S} 1$ and introducing a third human operator. A visual resume of the scenarios can be found in Figs. 7-9. In all the three scenarios, the users tried two modalities. In a 

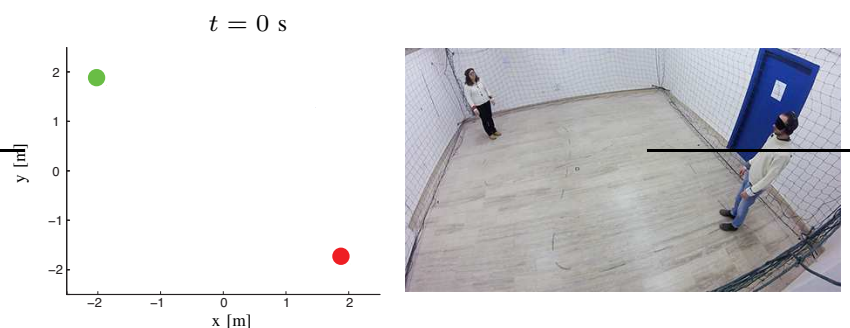

$t=9.3 \mathrm{~s}$
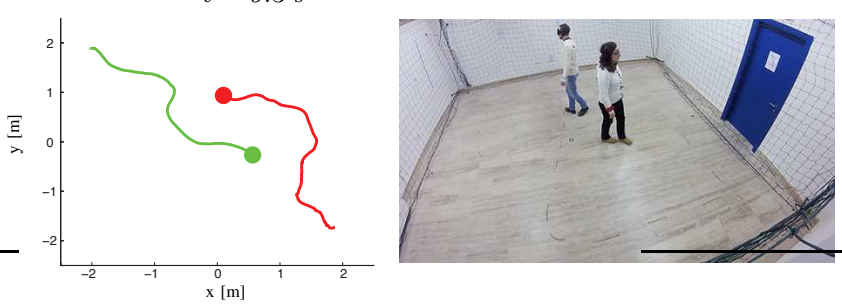

$t=14.8 \mathrm{~s}$
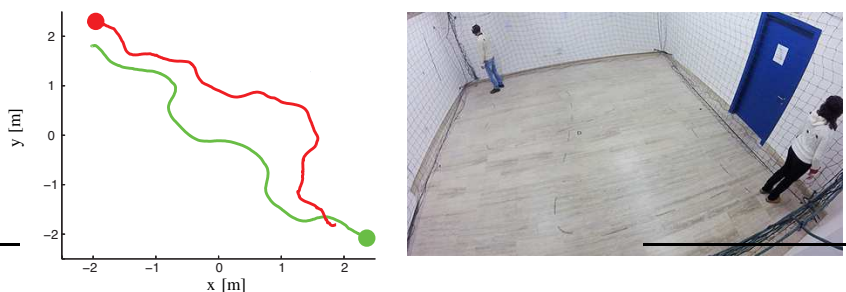

Fig. 7. Experimental validation for scenario S1. Two blindfolded and audiooccluded users have to move towards their goal areas by following directional cues provided by haptic armbands. (Left) The trajectories performed by the users are shown in red and green. The users are represented with colored circles. The starting point and the goal are two circles of radius $0.35 \mathrm{~m}$ centered in two opposite vertices of a square room with side of $2 \mathrm{~m}$. (Right) Snapshots of a performed trial. Haptic stimuli are provided to the user via two vibrotactile wristbands.

first modality $(\mathrm{H})$, users were blindfolded and were wearing earphones for masking the distinguishable sounds of the vibrotactile interfaces. In a second modality (V), users were able to see the environment and the other agents (other users or the obstacle), and no auditory occlusion was provided. In both modalities, users were allowed to choose their natural walking speed in order to perform the task. Each agent was modeled as a unicycle robot, pose and velocity were computed using an Extended Kalman Filter acquiring measures with a Vicon optical tracking system, composed of eight cameras. Six passive retro-reflective optical markers were placed on each user's torso, following the strong analogy between the steering wheels of a mobile robot and the human trunk, [14]. The sampling frequency of the motion capture system was set to $100 \mathrm{~Hz}$. In the experimental validation, we represented the users and the obstacles by their bounding circle; it is a common choice to represent objects by its bounding area or volume. The proposed evaluation was performed on 18 healthy subjects (twelve males, age range 23-49, 15 right-handed): one of them had great experience with the proposed vibro-tactile armbands, the remaining users had less or no experience with our haptic interfaces. None of the participants reported any deficiencies in perception abilities or physical impairments. The participants signed informed consent forms. All of them volunteered to the experiment, were informed about the purpose of the it, and were able to discontinue participation at any time. The motion control and related haptic stimulus was sent to the user
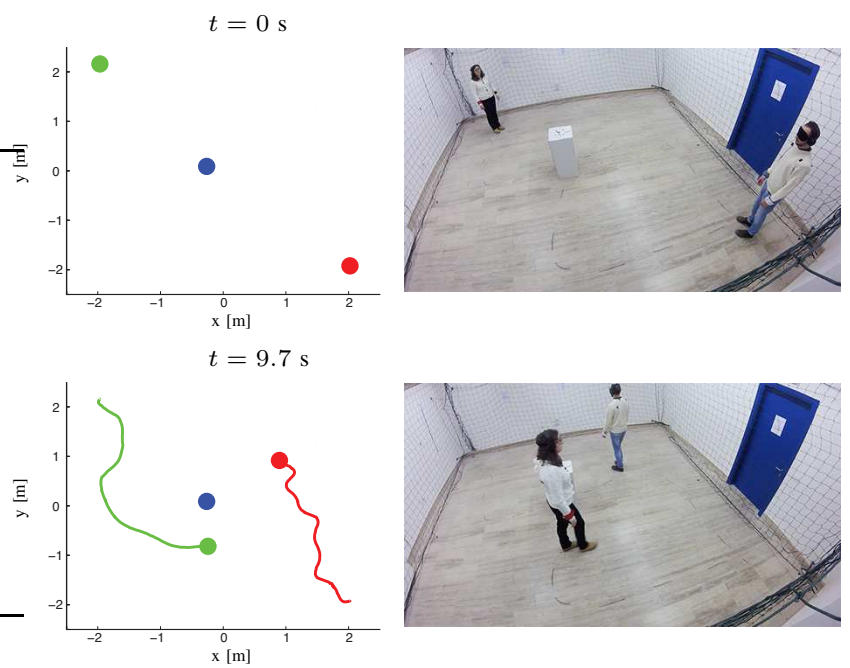

$t=19.3 \mathrm{~s}$
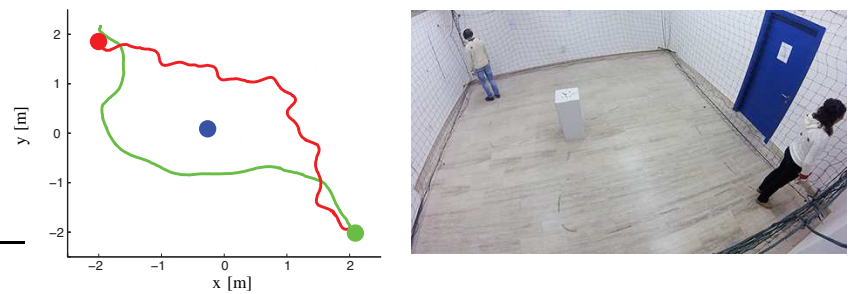

Fig. 8. Experimental validation for scenario S2. (Left) Two blindfolded and audio-occluded users have to move towards their goal areas, while avoiding a static obstacle. (Left) The trajectories performed by the users are shown in red and green, whereas the obstacle is depicted with a blue circle. The users are represented with red and green colored circles. The starting point and the goal are two circles of radius $0.35 \mathrm{~m}$ centered in two opposite vertices of a square room with side of $2 \mathrm{~m}$. (Right) Snapshots of a performed trial. Haptic stimuli are provided to the user via two vibrotactile wristbands.

each $0.2 \mathrm{~s}(5 \mathrm{~Hz})$ according to the duration of the vibro-tactile stimuli (cf. Sect. III-II).

The 18 participants, labeled from U1 to U18 for convenience, were divided per scenario as follows: all users, i.e., U1 to $\mathrm{U} 18$, performed four repetitions of scenario $\mathrm{S} 1$ and $\mathrm{S} 2$ per modality, being arranged in couples (U1 with U2, U3 with U4, etc.). For what concerns S3, trios/triplets of users were formed, being $\mathrm{U} 1, \mathrm{U} 2$, and $\mathrm{U} 3$ in a group, U4, U5, and U6 in another group, and so forth; For S3, four trials were performed per modality. Each user performed the trials in pseudo-randomized order. In each trial, users were asked to start from predefined positions and to reach predefined goals. The users walked in a square room with a side of $2 \mathrm{~m}$. The radius of the bounding circle was set to $0.35 \mathrm{~m}$ for each user. The starting point and the goal are two circles of radius $0.35 \mathrm{~m}$. For the scenario $\mathrm{S} 2$, in which users were asked to avoid a static obstacle, we represented the obstacle with a $0.3 \mathrm{~m}$ circle. The goal was considered successfully reached as soon as the center of the bounding circle of the user entered the goal area. A computer connected to the motion tracking system was in charge of: Acquiring human positions from the motion tracking system measurements; computing the optimal turning direction (or no turning); sending communication signals to the wearable haptic interfaces, for activating the vibro-tactile cues. 


\section{RESUlts AND Discussion}

Three metrics were used for evaluating the functionality of our approach: Time to reach the goal, length of the trajectories, and minimal distance from agents. Results on the recorded trials for these metrics are summarized in Fig. 10. In all trials and for all the modalities, no collision with other agentseither another user or the obstacle-has happened. While for the visual conditions this was expected, regarding the haptic guidance condition the obtained results show that our approach works, i.e., our system is able to successfully guide two or three users along collision-free paths, towards a goal area. Let us now analyze the obtained results.

In Fig. 10, data from modality $\mathrm{H}$ (where haptic suggestions were provided to the users) are depicted with blue bars, while green bars represent data from trial in modality $\mathrm{V}$ (subjects with no vision and audio impairment). Fig. 10(a) shows the completion trial for all three scenarios. As we can observe, users were taking less time to reach the goal-in all scenarios-while they were able to see the surrounding environment (modality V). This result is not surprising since it is known that human subjects slow down their walking pace when the visual feedback from the environment is removed, i.e., the vision channel is occluded. Fig. 10(b) presents the recorded trajectory lengths for the three scenarios. In this case, the difference between visual trials (modality V) and situations where the users were suggested by haptic cues is not as vast
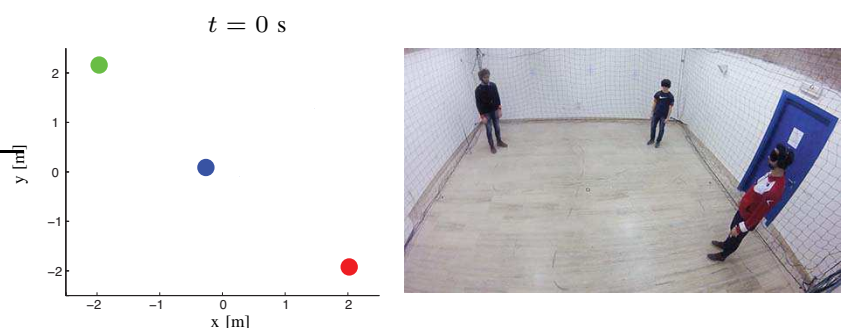

$t=6.2 \mathrm{~s}$
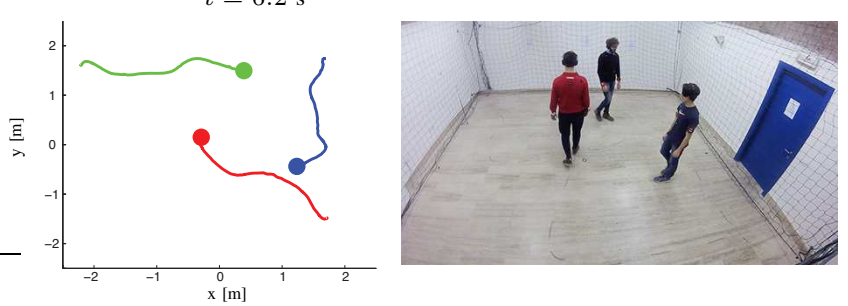

$t=14.8 \mathrm{~s}$
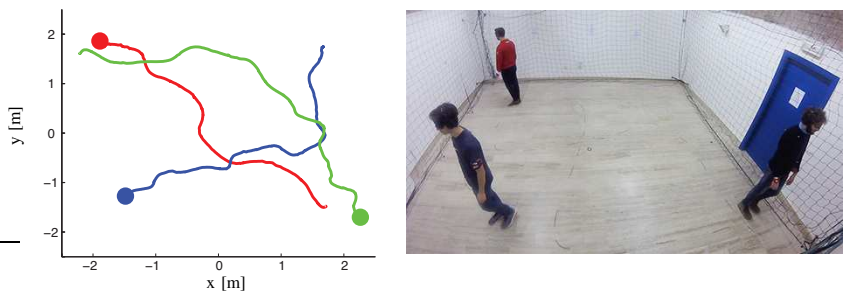

Fig. 9. Experimental validation for scenario S3. Three blindfolded and audiooccluded users are guided to reach the opposite corner of square room having side of $2 \mathrm{~m}$. (Left) The trajectories performed by the user are shown in red, green, and blue. The users are represented with colored circles. The starting point and the goal are two circles of radius $0.35 \mathrm{~m}$. (Right-handed sequence) Snapshots of a performed trial. Haptic stimuli are provided to the user via two vibrotactile wristbands. as for the time to reach the goal. Fig. 10(c) depicts the mean minimal distance (and standard deviation) between the agents recorded among all trials for a certain modality and a certain scenario. This last parameter can be seen as a measure on how much the system is daring in making people passing close. While it is clear that the system behaved similarly for the three scenarios in modality $\mathrm{H}$ (haptic suggestions were give to blindfolded, audio-occluded subjects), it is interesting to note how users were still passing fairly distant (between $1 \mathrm{~m}$ and $1.2 \mathrm{~m}$ ) in case of $\mathrm{S} 1$, and closer to each other (and the obstacle) for the other two scenarios. This behavior may be explained by the fact that a third known point (the obstacle for S2 and the third user for S3) can give a sort of augmented confidence to the user. However, this type of result will be analyzed in future works.

Comparison of the means among feedback conditions and scenarios was tested using a series of paired-samples t-tests. Data are mean \pm standard deviation, unless otherwise stated. Salient differences, where present, would like to be found on the same scenario, but among two different feedback conditions. For this reason, nine paired-samples t-tests were run, to check whether in the three different scenarios (S1, $\mathrm{S} 2$, and S3) the three metrics of interests (time to reach the goal, length of the walked path, and minimum distance among users) would prove to be more significant when users were given haptic cues w.r.t. when users were left without cues. Since each set of data (each bar of Fig. 10) was used only once in our analysis, it was not necessary to adjust the alpha value to avoid raising the probability of making a Type I error. Each test $t_{i j}$ is labelled after type of metric $(i=1$ as time to reach the goal, $i=2$ as length of the path, and $i=3$ as minimum distance between agents) and the $j^{\text {th }}$ scenario. In all tests, the assumption of normality of data was not violated, as assessed by Shapiro-Wilk's test $\left(p_{11}=.204\right.$, $p_{12}=.667, p_{13}=.569, p_{21}=.818, p_{22}=.259, p_{23}=.574$, $\left.p_{31}=.418, p_{32}=.567, p_{33}=.405\right)$. Participant were found to be faster (i.e., to take less time to reach the goal) when not stimulated with haptic cues in all scenarios $\left(t_{11}(71)=11.943\right.$, $p<0.001, t_{12}(71)=11.664, p<0.001, t_{13}(71)=10.238$, $p<0.001$ ), while the length of the walked path differed only when more than two agents (S2 and S3) were considered $\left(t_{21}(71)=0.713, p=0.478, t_{22}(71)=5.259, p<0.001\right.$, $\left.t_{23}(71)=4.061, p<0.001\right)$. A similar result has been found for the minimum distance between agents, meaning that the participant were going closer to each other when they could see (no haptic cues given) and when more than two agents (S2 and S3) were considered $\left(t_{31}(35)=0.955, p=0.346\right.$, $\left.t_{32}(35)=4.119, p<0.001, t_{33}(23)=7.745, p<0.001\right)$.

\section{CONCLUSIONS AND FUTURE WORK}

In this paper, we address the problem of guiding multiple users along collision-free paths in situations with poor/no visibility and reduced hearing capabilities. We consider a dynamic environment made by static obstacles and multiple moving users. Haptic feedback is used as an interesting way to provide directional information when audio and visual modalities are not available. Haptic stimuli are generated by a 


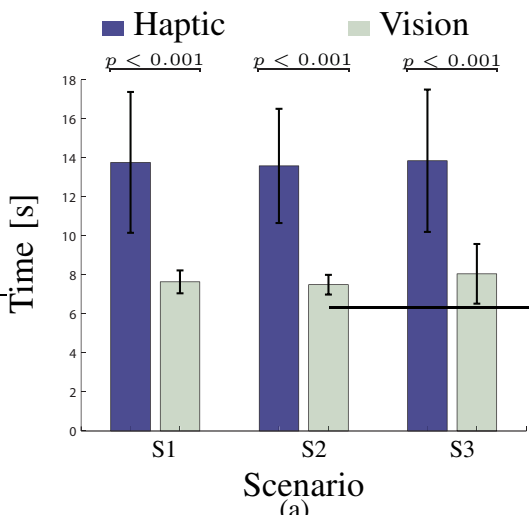

(a)

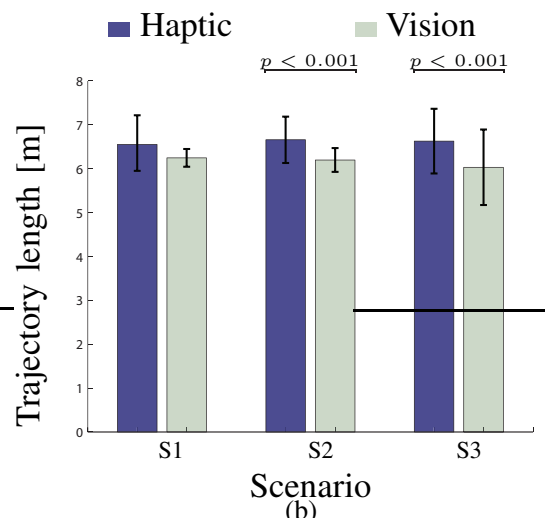

(b)

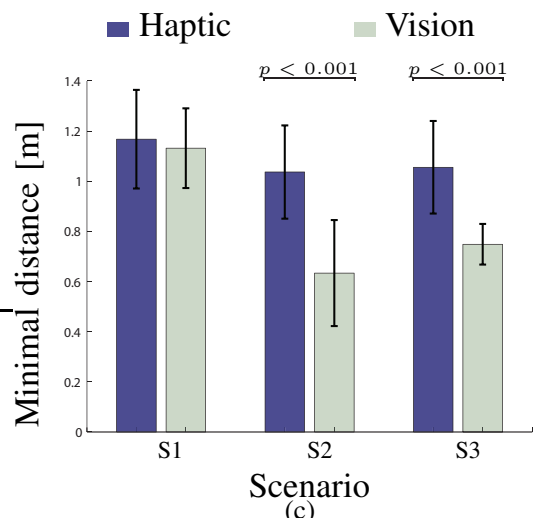

(c)

Fig. 10. Results of the experimental validation, divided by scenario: a) Mean time to reach the goal ( \pm standard deviation); b) mean length of the path ( \pm standard deviation) travelled; c) mean minimum distance between agents ( \pm standard deviation). Blue bars represent data where haptic suggestions were provided to the users $(\mathrm{H})$, whereas green bars represent data where visual information was provided to the users $(\mathrm{V})$. In (a) users took less time to reach the goal when they were able to see the surrounding environment (modality V) in all the cases. (b) The length of the performed trajectories is presented for the three scenarios. (c) The minimal distance recorded among all trials for a certain modality and a certain scenario is presented.

modified version of the Optimal Reciprocal Collision Avoidance algorithm for non-holonomic agents, which considers the reduced number of stimuli (i.e., instructions) that can be provided to the users and their motion uncertainty. The proposed navigation policy exploits the non-holonomic nature of human locomotion in goal directed paths, which leads to a very intuitive guidance mechanism. The proposed method is evaluated in three scenarios. Experimental results reveal that all the blindfolded subjects could safely reach the goal area. Although this result is promising, a comparison between the results obtained using this approach and experiments performed with sighted people reveals that additional studies need to be done in order to have comparable walking speed.

In future work, we will consider more challenging scenarios including the presence of narrow passages, and scenarios in which the users have a limited interval of time to accomplish the task. In this regard, we plan to evaluate the proposed approach in combination with haptic stimuli which suggest both the desired heading and pace to the user. Further studies will be performed to estimate online the way the users react to the guiding stimuli, in terms of linear and angular velocities. Finally, we plan to evaluate the proposed approach with different feedback modalities (composed of a different number of stimuli), and with older adults and visually impaired.

\section{REFERENCES}

[1] J. B. Van Erp, H. A. Van Veen, C. Jansen, and T. Dobbins, "Waypoint navigation with a vibrotactile waist belt," ACM Trans. on Applied Perception (TAP), vol. 2, no. 2, pp. 106-117, 2005.

[2] R. Lindeman, J. Sibert, R. Mendez-Mendez, S. Patil, and D. Phifer, "Effectiveness of directional vibrotactile cuing on a building-clearing task," in Proc. SIGCHI Conf. on Human factors in computing systems, 2005, pp. 271-280.

[3] A. Cosgun, E. Sisbot, and H. Christensen, "Guidance for human navigation using a vibro-tactile belt interface and robot-like motion planning," in Proc. IEEE Int. Conf. on Robotics and Automation, ICRA, 2014, pp. 6350-6355.

[4] M. Aggravi, S. Scheggi, and D. Prattichizzo, "Evaluation of a predictive approach in steering the human locomotion via haptic feedback," in Proc. IEEE/RSJ Int. Conf. Intelligent Robots and Systems, Hamburg, Germany, 2015.

[5] S. Scheggi, M. Aggravi, F. Morbidi, and D. Prattichizzo, "Cooperative human-robot haptic navigation," in Proc. IEEE Int. Conf. on Robotics and Automation, ICRA, 2014, pp. 2693-2698.
[6] S. Scheggi, M. Aggravi, and D. Prattichizzo, "Cooperative navigation for mixed human-robot teams using haptic feedback," IEEE Trans. on Human-Machine Systems, vol. 47, no. 4, pp. 462-473, 2017.

[7] M. Altini, E. Farella, M. Pirini, and L. Benini, "A cost-effective indoor vibrotactile navigation system for the blind." in HEALTHINF, 2011, pp. $477-481$.

[8] S. Bhatlawande, M. Mahadevappa, and J. Mukhopadhyay, "Way-finding electronic bracelet for visually impaired people," in Proc. IEEE Int. Conf. on Point-of-Care Healthcare Technologies, 2013, pp. 260-263.

[9] S. Bosman, B. Groenendaal, J.-W. Findlater, T. Visser, M. de Graaf, and P. Markopoulos, "Gentleguide: An exploration of haptic output for indoors pedestrian guidance," in Human-computer interaction with mobile devices and services, 2003, pp. 358-362.

[10] M. R. Adame, J. Yu, K. Moller, and E. Seemann, "A wearable navigation aid for blind people using a vibrotactile information transfer system," in Proc. Int. Conf. on Complex Medical Engineering, 2013, pp. 13-18.

[11] B. Sakcak, L. Bascetta, and G. Ferretti, "Human-like path planning in the presence of landmarks," in International Workshop on Modelling and Simulation for Autonomous Systems. Springer, 2016, pp. 281-287.

[12] P. Bevilacqua, M. Frego, E. Bertolazzi, D. Fontanelli, L. Palopoli, and F. Biral, "Path planning maximizing human comfort for assistive robots," in Proc. IEEE Int. Conf. on Control Applications, 2016, pp. 1421-1427.

[13] J. Alonso-Mora, A. Breitenmoser, M. Rufli, P. Beardsley, and R. Siegwart, "Optimal reciprocal collision avoidance for multiple nonholonomic robots," in Distributed Autonomous Robotic Systems, 2013, pp. 203-216.

[14] G. Arechavaleta, J.-P. Laumond, H. Hicheur, and A. Berthoz, "On the nonholonomic nature of human locomotion," Autonomous Robots, vol. 25, no. 1-2, pp. 25-35, 2008.

[15] S. Scheggi, F. Morbidi, and D. Prattichizzo, "Human-robot formation control via visual and vibrotactile haptic feedback," IEEE Trans. on Haptics, vol. 7, no. 4, pp. 499-511, 2014.

[16] L. Palopoli, A. Argyros, J. Birchbauer, A. Colombo, D. Fontanelli, A. Legay, A. Garulli, A. Giannitrapani, D. Macii, F. Moro, P. Nazemzadeh, P. Padeleris, R. Passerone, G. Poier, D. Prattichizzo, T. Rizano, L. Rizzon, S. Scheggi, and S. Sedwards, "Navigation assistance and guidance of older adults across complex public spaces: the dali approach," Intelligent Service Robotics, vol. 8, no. 2, pp. 77-92, 2015.

[17] S. Scheggi, A. Talarico, and D. Prattichizzo, "A remote guidance system for blind and visually impaired people via vibrotactile haptic feedback," in Control and Automation (MED), 2014 22nd Mediterranean Conf. of. IEEE, 2014, pp. 20-23.

[18] J. Van Den Berg, S. J. Guy, M. Lin, and D. Manocha, "Reciprocal nbody collision avoidance," in Robotics research. Springer, 2011, pp. 3-19.

[19] J. Van den Berg, M. Lin, and D. Manocha, "Reciprocal velocity obstacles for real-time multi-agent navigation," in Proc. IEEE Int. Conf. on Robotic and Automation, 2008, 2008, pp. 1928-1935.

[20] A. Riener, Sensor Actuator Supported Implicit Interaction in Driver Assistance Systems, 2010. 